\title{
Diagnostic de réseaux de Petri partiellement observables avec indicateurs algébriques
}

\section{Diagnosis of partially observed Petri nets with algebraic indicators}

\author{
Amira Chouchane ${ }^{1}$ et Philippe Declerck ${ }^{2}$ \\ ${ }^{1}$ RELEV, Ecole national d'ingenieurs de Sfax, Université de Sfax, Route de la Soukra km 4, 3038 sfax, Tunisia, \\ choucheneamira41@yahoo.fr \\ ${ }^{2}$ LARIS EA4094, University of Angers, 62 avenue Notre-Dame du Lac, 49000 Angers, France, \\ philippe.declerck@univ-angers.fr
}

\begin{abstract}
RÉSUMÉ. Dans cet article, nous présentons une approche de diagnostic d'un réseau de Petri partiellement observable basée sur une formalisation des indicateurs de fautes exprimées sous une forme algébrique (min, max, +). Les fautes sont modélisées par certaines transitions non observables et l'occurence d'une faute est équivalente au franchissement de la transition associée. L'algorithme d'élimination de Fourier-Motzkin est appliqué hors-ligne pour la génération des indicateurs de fautes utilisés pour le diagnostic en-ligne dans un temps polynomial. L'approche permet d'anticiper sur l'état du système (défaillant, non-défaillant, incertain) pour chaque transition observée de l'observation. Une comparaison avec la technique de diagnostic, utilisant la programmation linéaire, permet de montrer l'efficacité de notre approche. Le système de diagnostic se présente sous la forme d'un module opérant en parallèle avec l'approche classique.

ABSTRACT. In this paper, we present a diagnostic approach for partially observable Petri nets based on the formalization of fault indicators under the algebraic form $(\min , \max ,+)$. The faults are modeled by certain unobservable transitions, and the occurrence of a fault is equivalent to the firing of the associated transition. The Fourier-Motzkin elimination algorithm is applied off-line for the generation of fault indicators used on-line for fault diagnosis in a polynomial time. This approach allows anticipating the system state (faulty, no-faulty, uncertain) for each observed transition of the observation. A comparison with the diagnostic technique using the classical form of linear programming problems shows the effectiveness of our approach. The diagnostic system is a module operating in parallel with the classical approach.

MOTS-CLÉS. Diagnostic, réseau de Petri partiellement observable, indicateurs de fautes, Fourier-Motzkin, forme algébrique (min, $\max ,+$ ).

KEYWORDS. diagnosis, partially observed Petri net, fault indicators, Fourier-Motzkin, algebraic form (min, max, + ).
\end{abstract}

\section{Introduction}

Cet article porte sur le problème de diagnostic de systèmes qui peuvent être représentés par un Réseau de Petri (RdP). Les RdPs permettent en effet de modéliser des phénomènes de synchronisme, d'assemblage et de partage de ressources, présents dans de nombreuses applications comme les réseaux de transport, les systèmes informatiques et les systèmes multimédia. Un RdP pouvant contenir des transitions associées à des événements non observables. Ce réseau sera appelé RdP partiellement observable. Nous supposons que les fautes qui peuvent affecter le système sont des événements indésirables qui seront modélisés par les tirs de certaines transitions non observables.

L'objectif de cet article est de présenter le principe général d'un système de diagnostic pour les RdPs partiellement observables dont les indicateurs de fautes vont permettre de caractériser les fautes du processus après chaque occurrence d'un événement observé. Souvent, les approches alternant la détermination des vecteurs de franchissement et la génération des marquages, qui seront les points de départ aux futures estimations, conduisent à retarder l'émission d'états par le système de diagnostic. En effet, la taille de ces ensembles peut être importante, ce qui constitue une réelle difficulté.

Face à cette difficulté, notre but va être de réduire les énumérations de solutions tout en suivant les grandes lignes de l'approche [CABASINO et al. 2010]. Partant du corollaire 3.9 dans [CABASINO et al. 
2010], nous approfondissons cette étude en exprimant les indicateurs de fautes sous forme de problèmes de la programmation linéaire. Nous proposons aussi une adaptation de l'algorithme de Fourier-Motzkin dont l'intérêt est d'exprimer explicitement sous une forme algèbrique les liens existant entre d'un côté, les occurrences des fautes et de l'autre, les observations et le marquage courant. Le diagnostic pourra alors être conçu en deux phases. La première phase est un pré-calcul hors-ligne qui n'est effectué qu'une seule fois. Elle contient la partie coûteuse en temps de l'algorithme d'élimination de Fourier-Motzkin. La seconde phase est une procédure en-ligne qui exploite les données courantes et met en valeur les fautes potentiels. Elle permet de générer les indicateurs permettant l'interprétation de l'état du système en se basant uniquement sur l'ensemble des marquages possibles ceci de manière polynomiale. Il est important de noter qu'elle ne nécessite pas la détermination des vecteurs d'explications qui seront calculés après dans l'itération courante. Ainsi, même si l'approche classique alternant estimation des vecteurs d'explications et des marquages est identique, la procédure de diagnostic pourra de manière anticipée générer l'état du système : cette anticipation pourra être cruciale dans le cas d'une panne catastrophique.

Sans être exhaustif, nous présentons maintenant quelques travaux remarquables de la littérature. En raison de l'explosion combinatoire des états, les approches [JIROVEANU et al. 2008] et [CABASINO et al. 2010] sont basées sur un observateur réduit qui ne calcule qu'un sous-ensemble des marquages possibles pour un RdP partiellement observable. L'étude [RU et al. 2009] montre que la complexité de l'estimation du nombre de marquages estimés est polynomiale pour la longueur de la séquence observée sous l'hypothèse d'un RdP borné. La technique de fluidification présentée dans [MAHULEA et al. 2012] et [CHOUCHANE et al. 2018] peut être une solution pour réduire l'explosion combinatoire lorsque le marquage initial est grand. Une augmentation de la rapidité de diagnostic se fera cependant au détriment d'une baisse de précision des résultats. Dans l'esprit des trois états de diagnostic (N, U, F) de [MAHULEA et al. 2012] qui se distinguent des quatres états (de 0 à 4) de [CABASINO et al. 2010], nous proposons différentes écritures des indicateurs de fautes qui limiteront l'aspect énumératif en évitant de traiter l'ensemble des vecteurs d'explications minimaux mais en choisissant des vecteurs optimaux en posant différents problèmes de la programmation linéaire en nombres entiers. Contrairement à [CABASINO et al. 2010], chaque vecteur d'explication sera relatif à deux observations successives. L'élimination de Fourier-Motzkin est aussi utilisée dans [AHMED et al. 2016] où deux ensembles d'inégalités pour chaque transition de faute sont déduits mais sans cependant exploiter la condition de franchissement des transitions observables et une formalisation des matrices observable et non-observable contrairement à notre approche. Une autre différence est aussi que l'approche [AHMED et al. 2016] ne suit pas le principe de l'alternance entre la génération des vecteurs de franchissement et la production des marquages. L'article [LEFEBVRE 2012] traite un problème général considérant des événements et des marquages partiellement connus, donne des conditions complémentaires pour limiter le nombre de séquences à traiter et formalise la détection de l'état défaillant.

Nous pouvons aussi citer les approches de diagnostic basées sur la construction hors-ligne de graphes utilisés pour le diagnostic en-ligne des RdPs. Dans [CABASINO et al. 2013], il est montré que si le RdP étiqueté est borné, la construction hors-ligne d'un automate appelé graphe d'accessibilité de base (BRG) est possible, utilisé pour un diagnostic en-ligne. Un BRG étendu (EBRG) a été construit dans [RAN et al. 2017] qui est un automate basé sur des marquages de base calculés en supposant que toutes les fautes du système sont observables. Géneralement, le EBRG admet beaucoup moins de noeuds que le graphe d'accessibilité, mais ce nombre reste important. Dans [BASILE et al. 2014], les auteurs s'intéressent au diagnostic d'un RdP temporel (TPN) étiqueté partiellement observable. Un algorithme est présenté pour 
la construction hors-ligne d'un graphe appelé graphe de classe d'état modifié (MSCG) inspiré du graphe de classes d'état (SCG) introduit dans [BERTHOMIEU et al. 1991], qui recueille toutes les informations sur l'évolution du système. Une procédure en-ligne est introduite qui, à partir d'une observation temporisée, c'est-à-dire une séquence d'étiquettes avec leurs instants de tir et un instant d'estimation $\tau$, permet de déterminer dans quel état le système peut être à $\tau$ en utilisant le MSCG et en résolvant un certain nombre de problèmes de programmation linéaire pour le diagnostic de TPN. Dans [SUN et al. 2014], les auteurs s'intéressent au diagnostic d'un RdP étiqueté partiellement observable. Le diagnostic en-ligne est résolu en se basant sur une construction incrémentale et à-la-volée de deux structures en parallèle, appelées respectivement le graphe de marquage de faute (FMG) et le graphe de l'ensemble de marquage de faute (FMSG). Dans [BOUSSIF et al. 2017], une approche de diagnostic est basée sur la construction hors-ligne d'un graphe appelé diagnostiqueur semi-symbolique (SSD). Le RdP est supposé sans blocage, borné et avec un sous-réseau non observable acyclique. Les fautes sont modélisées par des transitions non observables et supposées être permanentes. L'approche de diagnostic en-ligne est basée sur le SSD, calculé à-la-volée, selon lequel ces noeuds sont codés à l'aide de diagrammes de décision binaires (BDD) et qui sont reliés par des arcs étiquetés par des transitions. Chaque noeud dans le SSD est partitionné en deux sous-ensembles distincts de marquages : l'ensemble de marquages normaux, qui est l'ensemble de marquages atteignables en franchissant des séquences non défaillantes, et l'ensemble de marquages défectueux, qui est l'ensemble des marquages atteignables en franchissant des séquences défaillantes.

Dans cet article, on suppose que la matrice d'incidence et le marquage initial du RdP sont connus. Les occurrences de franchissement des transitions observables sont supposées non simultanées. Nous prenons l'hypothèse de non cyclicité du sous réseau non-observable qui assure que les solutions trouvées par la méthode algébrique correspondent à des séquences de franchissement réelles de transitions non observables (STREMERSCH et BOEL 2002). Pour le diagnostic, chaque faute sera relative à une fenêtre glissante élémentaire encadrée par deux observations successives. Une généralisation sur un horizon quelconque sera présentée prochainement. Chaque espace de solutions considéré est supposé non vide et est borné quelles que soient les observations considérées. On suppose de plus que toute trajectoire pourra toujours être prolongée en accord avec de nouvelles observations.

L'article suit le plan suivant. Après avoir donné les préliminaires dans la section 2, nous présentons le principe de l'approche proposée dans la section 3. Dans la section 4 , nous décrivons un modèle de la forme $A . x \leq b$ qui décrit l'évolution du système pour une itération. Section 5 présente la procédure horsligne/en-ligne basée sur l'algorithme de Fourier-Motzkin et les indicateurs de fautes. Dans la section 6, un benchmark permet de montrer l'efficacité numérique des approches proposées et de les comparer.

\section{Préliminaires}

La notation $|Z|$ exprime le cardinal de l'ensemble $Z$ et la notation $A^{T}$ correspond à la matrice $A$ transposée. Un RdP est un graphe du type Place/Transition (P/TR) défini par la structure $N=\left(P, T R, W^{+}, W^{-}\right)$, où $P$ est un ensemble de $|P|$ places et $T R$ est un ensemble de $|T R|$ transitions. Les matrices $W^{+}$ et $W^{-}$sont respectivement les matrices $|P| \times|T R|$ post et pré-incidentes sur $\mathbb{N}$, où chaque ligne $l \in\{1, \ldots,|P|\}$ précise le poids des arcs entrants et sortants de la place $p_{l} \in P$. Un RdP étiqueté est un réseau $\left(P, T R, W^{+}, W^{-}\right)$avec la fonction d'étiquetage $\ell: T R \rightarrow L \bigcup\{\varepsilon\}$ qui associe à chaque 
transition $t \in T R$ soit un symbole d'un alphabet donné $L$, soit la chaîne vide $\varepsilon$.

Dans un RdP partiellement observable, une transition est dite observable (respectivement, non-observable) si chaque tir de cette transition est un événement connu (respectivement, inconnu) pour le système d'estimation observant le RdP. L'ensemble $T R_{o b}$ (respectivement, $T R_{n o}$ ) est l'ensemble des transitions observables dont l'étiquette est dans $L$ (respectivement, non-observables dont l'étiquette est $\varepsilon$ ) et $T R=T R_{o b} \bigcup T R_{n o}$. Le sous-graphe induit $T R_{n o}$ (respectivement $T R_{o b}$ ) du réseau de Petri $N$ est défini comme un nouveau graphe $N_{n o}=\left(P, T R_{n o}, W_{n o}^{+}, W_{n o}^{-}\right)$(respectivement $N_{o b}=\left(P, T R_{o b}, W_{o b}^{+}, W_{o b}^{-}\right)$), où $W_{n o}^{+}$et $W_{n o}^{-}$(respectivement, $W_{o b}^{+}$and $W_{o b}^{-}$) sont des restrictions de $W^{+}$et $W^{-}$à $P \times T R_{n o}$ (respectivement, $P \times T R_{o b}$ ). Ainsi, $W_{n o}=W_{n o}^{+}-W_{n o}^{-}$(respectivement, $\left.W_{o b}=W_{o b}^{+}-W_{o b}^{-}\right)$. Nous supposons que la matrice d'incidence $W$ de $N$ est égale à $\left(\begin{array}{ll}W_{n o} & W_{o b}\end{array}\right)$ ce qui est obtenu après un éventuel classement des colonnes selon $T R_{n o}$ et $T R_{o b}$. La notation $x_{i}$ décrit une transition non-observable, appartenant à $T R_{n o}$ tandis qu'une transition observable appartenant à $T R_{o b}$ est notée $y_{i}$.

Une séquence de franchissements est le franchissement successif de transitions dans un ordre donné à partir d'un marquage donné. Nous notons $T R^{*}$ l'ensemble des séquences de franchissements. Cette définition est étendue à $T R_{o b}^{*}$ et $T R_{n o}^{*}$. Un mot observé, noté $w$, est une succession de labels associés à des transitions observées. Un mot est vide s'il n'existe pas des transitions observées. Le vecteur $\bar{\sigma}$ (respectivement, $\bar{x}$ et $\bar{y}$ ) de dimension $|T R|$ (respectivement, $\left|T R_{n o}\right|$ et $\left|T R_{o b}\right|$ ) exprime le vecteur de franchissement de la séquence $\sigma \in T R^{*}$ (respectivement, $x \in T R_{n o}^{*}$ et $y \in T R_{o b}^{*}$ ), où le $i$-th composant $\overline{\sigma_{i}}$ (respectivement, $\overline{x_{i}}$ et $\overline{y_{i}}$ ) est le nombre de tirs de la transition $t_{i} \in T R$ (respectivement, $x_{i} \in T R_{n o}$ et $\left.y_{i} \in T R_{o b}\right)$. Nous avons $\bar{\sigma}=\left(\begin{array}{cc}\bar{x}^{T} & \bar{y}^{T}\end{array}\right)^{T}$.

Le marquage de l'ensemble $P$ est un vecteur $M \in \mathbb{N}^{|P|}$ qui affecte à chaque place $p_{i} \in P$ un nombre entier non-négatif de jetons $M_{i}$. La $i$-ème composante $M_{i}$ est aussi écrite $M\left(p_{i}\right)$. Le marquage $M$ atteint à partir du marquage initial $M^{\text {init }}$ (qui remplace la notation usuelle $M_{0}$ qui n'est pas cohérente avec la notation mathématique habituelle des vecteurs) par le tir de la séquence $\sigma$ peut être calculé avec la relation fondamentale : $M=M^{i n i t}+W . \bar{\sigma}$. La transition $\operatorname{tr}$ est tirable pour $M$ si $M \geq W^{-}(., t r)$ et son tir effectif conduit au marquage $M^{\prime}=M+W(., t r)$. Nous écrivons $M[\sigma \succ$ pour exprimer que la séquence de transitions $\sigma$ est tirable à $M$, et nous écrivons $M\left[\sigma \succ M^{\prime}\right.$ pour décrire que le tir de $\sigma$ conduit à $M^{\prime}$.

\section{Formulation du problème et principe général de l'approche}

Le problème considéré dans cet article est le suivant. Nous considérons un RdP où la matrice d'incidence $W$ et le marquage initial $M^{\text {init }}$ sont connus. Compte tenu d'une séquence de franchissement observée $y \in T R_{o b}^{*}$ générée par l'évolution du RdP, nous voulons caractériser les séquences non observables $x \in T R_{n o}$ qui sont cohérents avec l'observation $y$.

Le principe de l'approche est basé sur le traitement des données produites successivement par les transitions de la séquence observée $y$ dans une procédure en-ligne [CABASINO et al. 2010] [JIROVEANU et al. 2008]. Soit $y=y^{<1>} \ldots y^{<h>}$ où $y^{<k>}$ est le $k^{\text {ième }}$ transition observée de $y$ et $h=\operatorname{card}(y)$ est la longueur de l'observation. Alors, à chaque itération $\langle k\rangle$, il existe des séquences non observables possibles qui permettent le tir de $y^{<k>}$. Le franchissement d'une séquence non-observable possible, notée $x^{<k>}$ et la transition observée $y^{<k>}$ génèrent un nouveau marquage utilisé comme marquage initial pos- 
sible dans l'itération suivante. L'équation fondamentale de marquage du RdP est une fonction linéaire du marquage initial et des vecteurs de franchissement des séquences observables et non observables. Alors, pour un RdP à structure connue et si on observe le franchissement d'une séquence à partir d'un marquage initial connu, il suffit d'estimer les vecteurs de franchissement des séquences non observables possibles cohérentes avec la séquence observée pour déduire les marquages actuels possibles du RdP.

La procédure de recherche est faite successivement pour la transition observée $y^{<1>}$ jusqu'à la transition observée $y^{<h>}$. Finalement, si nous considérons une séquence unique à chaque itération, nous obtenons :

$$
\begin{array}{ll}
M^{<1>}\left[x^{<1>}\right. & \succ M^{\prime} \text { avec } M^{\prime}\left[y^{<1>} \succ M^{<2>}, M^{<2>}\left[x ^ { < 2 > } \succ M ^ { \prime \prime } \text { avec } M ^ { \prime \prime } \left[y^{<2>} \succ M^{<3>}, \ldots\right.\right.\right. \\
\ldots M^{<h>}\left[x^{<h>}\right. & \succ M^{\prime \prime \prime} \text { avec } M^{\prime \prime \prime}\left[y^{<h>} \succ M^{<h+1>}\right.
\end{array}
$$

où $M^{<1>}=M^{\text {init }}$. Alors, le dernier marquage $M^{<h+1>}$ est un marquage actuel possible cohérent avec l'observation $y$ à partir de $M^{\text {init }}$. Tous les marquages possibles sont ceux obtenus en développant toutes les séquences de franchissement possibles $\left\{x^{<1>}, x^{<2>}, \ldots, x^{<h>}\right\}$. Lorsqu'un vecteur de franchissement correspond à une séquence qui peut être suivie par le RdP, ce vecteur de franchissement est appelé vecteur d'explication [CABASINO et al. 2010] et est défini pour chaque itération $\langle k\rangle$ de la manière suivante :

DEFINITION 2..1- Soit $x^{<k>}$ une séquence de franchissement non-observable qui conduit au franchissement de la transition observée $y^{<k>}$ à partir d'un marquage donné $M^{<k>}$ pour l'itération $<k>$. Un vecteur d'explication de $y^{<k>}$ à partir de $M^{<k>}$ est le vecteur de franchissement de $x^{<k>}$ noté $\overline{x^{<k>}}$. Les ensembles des séquences non observables possibles et des vecteurs d'explication pour le couple $\left(M^{<k>}, y^{<k>}\right)$ sont désignés respectivement par $S E Q\left(M^{<k>}, y^{<k>}\right)$ et $E\left(M^{<k>}, y^{<k>}\right)$. Formellement,

$$
\begin{aligned}
& S E Q\left(M^{<k>}, y^{<k>}\right)=\left\{x^{<k>} \mid x^{<k>} \in T R_{n o}, M^{<k>}\left[x^{<k>} \succ M^{\prime} \text { et } M^{\prime}\left[y^{<k>} \succ\right\}\right.\right. \\
& E\left(M^{<k>}, y^{<k>}\right)=\left\{\overline{x^{<k>}} \mid x^{<k>} \in S E Q\left(M^{<k>}, y^{<k>}\right\}\right.
\end{aligned}
$$

pour l'itération $\left\langle k>\right.$. L'ensemble des vecteurs d'explication noté $E^{<k>}$ à l'itération $<k>$ est l'union des ensembles $E\left(M^{<k>}, y^{<k>}\right)$ qui sont obtenus en considérant chaque marquage initial possible $M^{<k>} \in \mathcal{M}^{<k>}$, soit

$$
E^{<k>}=\bigcup_{M<k>\in \mathcal{M}<k>} E\left(M^{<k>}, y^{<k>}\right)
$$

Tous les franchissements de transitions dans $x^{<k>}$ ne sont pas indispensables au franchissement de $y^{<k>}$. Parmi toutes ces séquences, il est intéressant de distinguer celles représentant un fonctionnement strictement nécessaire : le vecteur de franchissement est alors minimal. Contrairement aux vecteurs maximaux, les vecteurs minimaux existent toujours car $\overline{x^{<k>}} \geq 0$.

$$
\begin{aligned}
& S E Q^{\min }\left(M^{<k>}, y^{<k>}\right)=\left\{x^{<k>} \in S E Q\left(M^{<k>}, y^{<k>}\right) \mid \nexists x^{\prime<k>} \in S E Q\left(M^{<k>}, y^{<k>}\right)\right. \\
& \left.\operatorname{avec} \overline{x^{\prime<k>}} \leq \overline{x^{<k>}} \text { et } \overline{x^{\prime<k>}} \neq \overline{x^{<k>}}\right\} \\
& E^{\min }\left(M^{<k>}, y^{<k>}\right)=\left\{\overline{x^{<k>}} \mid x^{<k>} \in S E Q^{\min }\left(M^{<k>}, y^{<k>}\right\}\right.
\end{aligned}
$$

pour l'itération $\left\langle k>\right.$. Notons que le marquage $M^{<k>}$ est déduit à l'itération $<k-1>$ mais est utilisé comme une donnée connue à l'itération $<k>$ comme l'observation $y^{<k>}$. De manière cohérente, l'ensemble des marquages calculés à l'itération $<k>$ mais utilisés à l'itération $<k+1>$ est noté 
$\mathcal{M}^{<k+1>}$ et est déterminé par la considération de tous les marquages $M^{<k>} \in \mathcal{M}^{<k>}$ (obtenus à l'itération $\langle k-1\rangle$ ) et des séquences correspondantes calculées à l'itération $\langle k\rangle$. Plus formellement, l'ensemble des marquages de départ pour l'itération $<k+1>$ est défini itérativement :

$$
\begin{aligned}
& \mathcal{M}^{<k+1>}=\left\{M^{<k+1>} \in \mathbb{N}^{|P|} \mid\left(\exists M^{<k>} \in \mathcal{M}^{<k>}\right),\left(\exists x^{<k>} \in S E Q\left(M^{<k>}, y^{<k>}\right)\right),\right. \\
& M^{<k>}\left[x^{<k>} \succ M^{\prime} \text { et } M^{\prime}\left[y^{<k>} \succ M^{<k+1>}\right\} \text { pour } k \geq 1 \text { avec } \mathcal{M}^{<1>}=\left\{M^{<1>}\right\}\right.
\end{aligned}
$$

De même, l'ensemble des marquages de base [CABASINO et al. 2010] pour l'itération $<k+1>$ est un sous-ensemble de $\mathcal{M}^{<k+1>}$ défini comme suit :

$$
\begin{aligned}
& \mathcal{M}^{\min ,<k+1>}=\left\{M^{<k+1>} \in \mathbb{N}^{|P|} \mid\left(\exists M^{<k>} \in \mathcal{M}^{\min ,<k>}\right),\left(\exists x^{<k>} \in S E Q^{\min }\left(M^{<k>}, y^{<k>}\right)\right),\right. \\
& M^{<k>}\left[x^{<k>} \succ M^{\prime} \text { et } M^{\prime}\left[y^{<k>} \succ M^{<k+1>}\right\} \text { pour } k \geq 1 \text { avec } \mathcal{M}^{\min ,<1>}=\left\{M^{<1>}\right\}\right.
\end{aligned}
$$

\section{Polyèdre des vecteurs de franchissement}

Dans cette partie, nous considerons le franchissement d'une seule transition de l'observation. Si on observe le franchissement d'une transition $y^{<k>}$ pour un marquage de départ $M^{<k>} \in \mathcal{M}^{<k>}$, alors il existe une séquence de franchissement non-observable $x^{<k>}$ et un marquage $M^{\prime}$ tel que $M^{<k>}\left[x^{<k>} \succ\right.$ $M^{\prime}$ et $M^{\prime}\left[y^{<k>} \succ\right.$. Ainsi, $M^{\prime}$ est le marquage atteignable à partir du marquage $M^{<k>}$ en franchissant la séquence non-observable $x^{<k>}$ et ce marquage $M^{\prime}$ permet d'effectuer le franchissement de la transition observée $y^{<k>}$.

Comme $M^{<k>}\left[x \succ M^{\prime}\right.$, alors le marquage $M^{\prime}$ satisfait l'équation suivante :

$$
M^{\prime}=M^{<k>}+W_{n o} \cdot \bar{x}
$$

où $W_{n o}$ est la matrice d'incidence du sous réseau non-observable. Pour alléger l'écriture, on note $\overline{x^{<k>}}=$ $\bar{x}$. En outre $M^{\prime}\left[y^{<k>} \succ\right.$, alors nous pouvons écrire l'inégalité suivante :

$$
M^{\prime} \geq W_{o b}^{-}\left(., y^{<k>}\right)
$$

En remplaçant $M^{\prime}$ par son expression [1] de cette section et en considerant la contrainte de positivité $\bar{x} \geq 0$, le vecteur $\bar{x}$ doit alors vérifier l'inégalité matricielle suivante :

$$
A \cdot \bar{x} \leq b^{<k>}
$$

$\operatorname{avec} A=\left(\begin{array}{c}-W_{n o} \\ -I_{n \times n}\end{array}\right), \bar{x}=\overline{x^{<k>}}, b^{<k>}=\left(\begin{array}{c}M^{<k>}-W_{o b}^{-}\left(., y^{<k>}\right) \\ 0_{n \times 1}\end{array}\right)$ et $M^{<1>}=M^{i n i t}$. $A$ est une matrice de dimension $m \times n$ avec $n=\left|T R_{n o}\right|$ et $m=|P|+n$. Le nombre de lignes $m$ peut être réduit si nous supprimons les lignes nulles qui correspondent aux places non connectées à une transition non-observable. Contrairement au vecteur $b^{<k>}$, la matrice $A$ ne dépend pas de l'itération $\langle k>$.

\section{Fautes et indicateurs}

Dans cette section, nous considérons le diagnostic de fautes qui sont représentées par le tir de transitions qui sont non observables et l'objectif va être de détecter ces fautes. Comme naturellement il peut y avoir 
aussi d'autres transitions non observables qui représentent des comportements normaux, ceci peut être modélisé sous la forme d'un partitionnement de l'ensemble des transitions non observables sous la forme

$$
T R_{n o}=T R_{f} \cup T R_{n}
$$

où $T R_{f}$ est l'ensemble des transitions non observables dont le tir de chaque tranition exprime une faute et $T R_{n}$ est l'ensemble des transitions non observables qui représentent des comportements normaux.

Dans cet article, nous allons associer un ensemble de critères pour chaque faute apparaissant à l'itération $\left\langle k>\right.$, c'est à dire sur 1'horizon relatif aux observations $y^{<k-1>}$ et $y^{<k>}$ (permis par le franchissement préalable de $y^{<k-1>}$ et conduisant au franchissement de $y^{<k>}$ ). Par hypothèse de travail, les interprétations du diagnostic seront effectuées donc uniquement à partir de l'observation $y^{<k-1>}$ donnant $M^{<k>}$ et sans que le franchissement de $y^{<k>}$ soit un préalable à des franchissements de transitions non-observables. En raison du nombre limité de pages, la généralisation de ce point ainsi que la considération des classes de fautes pouvant contenir plusieurs transitions de faute seront présentées dans un autre article.

Dans le but de déterminer les états défaillants et non-défaillants des transitions de fautes, nous allons dans un premier temps utiliser la programmation linéaire qui ne nécessite pas la génération préalable de l'ensemble des vecteurs d'explication $E^{<k>}$ pour l'itération $\langle k>$.

\subsection{Formulation avec la programmation linéaire}

Les résultats suivants montrent que l'ensemble correspondant aux marquages de base $\mathcal{M}^{\text {min, }<k>} \subset \mathcal{M}^{<k>}$ sont des supports au diagnostic car ils permettent d'exprimer l'ensemble des marquages accessibles noté $\mathcal{C}^{<k>}$ pour l'observation du mot $y^{<1>} y^{<2>} \ldots y^{<k-1>} \in T R_{o b}^{*}$ ce qui est cohérent avec la notation des marquages $M^{<k>} \in \mathcal{M}^{<k>}$ produits à l'itération $<k-1>$ et utilisés à l'itération $<k>$.

Corollaire 1 - (corollaire 3.9 dans CABASINO, GIUA et SEATZU 2010) Soit un RdP dont le sousréseau non-observable est acyclique. Pour l'itération $<k>$,

$$
\mathcal{C}^{<k>}=\left\{M \in \mathbb{N}^{|P|} \mid\left(M=M^{<k>}+W_{n o} \cdot \bar{x}\right)\left(\forall \bar{x} \in \mathbb{N}^{n}\right)\left(\forall M^{<k>} \in \mathcal{M}^{\min ,<k>}\right)\right\} .
$$

Considèrant maintenant l'observation $y^{<k>}$, nous introduisons le corollaire suivant qui va permettre d'établir des indicateurs dont la détermination sera plus efficace.

COROllaire 2- Soit un RdP dont le sous-réseau non-observable est acyclique. Chaque vecteur $\bar{x} \in$ $\mathbb{N}^{n}$ dans [3] de la section 3 est un vecteur d'explication pour chaque marquage de base $M^{<k>} \in$ $\mathcal{M}^{\text {min, }<k>}$ à l'itération $<k>$.

Démonstration. Si maintenant, nous ajoutons au corollaire 1 une condition de franchissement $M\left[y^{<k>} \succ\right.$ à l'expression ci-dessus $\mathcal{C}(y)$, nous restreignons l'espace et nous retrouvons le raisonnement conduisant à l'établissement de [3] de la section 3 mais avec $M^{<k>} \in \mathcal{M}^{\text {min, }<k>}$

Il suffit ainsi de prendre tous les marquages de base au lieu des marquages de départ dans [3] de la section 3 ce qui est un premier moyen de réduire l'ensemble à considérer. 
Pour l'itération $\left\langle k>\right.$, on peut définir un premier indicateur de faute $J^{-}\left(x_{l},<k>\right)$ associé à chaque transition de faute $x_{l} \in T R_{f}$ qui, dans une vision "optimiste" sur l'état du système, exprime le nombre minimal d'occurrences de faute pour une observation $y^{<k>}$ :

$$
J^{-}\left(x_{l},<k>\right)=\min \left\{\overline{x_{l}}\left(\forall \bar{x} \in E^{<k>} \text { et } \forall M^{<k>} \in \mathcal{M}^{\min ,<k>}\right)\right\} \text { avec } x_{l} \in T R_{f}
$$

Ainsi, si $J^{-}\left(x_{l},<k>\right) \geq 1$ pour $x_{l} \in T R_{f}$, nous pouvons affirmer que la faute correspondant à $x_{l}$ a été détectée à l'itération $\langle k>$. Il exprime la localisation de cette faute (ou son isolation selon le vocabulaire utilisé en détection de défaillances pour les systèmes continus). De plus la situation $J^{-}\left(x_{l},<k>\right)>1$ garantit un caractère répété de la faute associée à $x_{l}$.

Symétriquement, nous pouvons prendre une vision "pessimiste" sur l'état du système et définir une fréquence maximale relative à chaque transition de faute $x_{l} \in T R_{f}$.

$$
J^{+}\left(x_{l}, \quad<k>\right)=\max \left\{\overline{x_{l}}\left(\forall \bar{x} \in E^{<k>} \text { et } \forall M^{<k>} \in \mathcal{M}^{\min ,<k>}\right)\right\} \text { avec } x_{l} \in T R_{f}
$$

Ainsi, si $J^{+}\left(x_{l},<k>\right)=0$ pour $x_{l} \in T R_{f}$, nous pouvons affirmer qu'aucune faute correspondant à $x_{l}$ n'a été détectée à l'itération $<k>$ par le système de diagnostic. Si $J^{+}\left(x_{l},<k>\right) \geq 1$, la faute a pu arriver avec le nombre d'occurrences $J^{+}\left(x_{l},<k>\right.$ ) au plus (mais éventuellement n'est pas arrivé si $\left.J^{-}\left(x_{l},<k>\right)=0\right)$.

En résumé, pour une transition de faute $x_{l}$ et l'itération $\langle k>$, nous pouvons affirmer de manière générale que la faute correspondant apparaît avec une fréquence comprise entre au moins $J^{-}\left(x_{l},<k>\right.$ ) et au plus $J^{+}\left(x_{l},<k>\right)$ avec $0 \leq J^{-}\left(x_{l},<k>\right) \leq J^{+}\left(x_{l},<k>\right)$. Plus particulièrement, nous pouvons déduire que :

1. Si $J^{-}\left(x_{l},<k>\right) \geq 1$ (c'est à dire $\overline{x_{l}} \geq 1$ apparaît pour tous les vecteurs d'explication), alors une faute $x_{l}$ est détectée. Cette situation est notée par $D$ comme Faute Détectée.

2. Si $J^{-}\left(x_{l},<k>\right)=0$, et si en plus, $J^{+}\left(x_{l},<k>\right)=0$ (c'est à dire aucun vecteur $\bar{x} \in E^{<k>}$ ne présente $\overline{x_{l}} \neq 0$ ) alors la procédure ne détecte aucune faute pour $x_{l}$. Cette situation est notée par $N$ comme Normal.

3. Si $J^{-}\left(x_{l},<k>\right)=0$, et si de plus, $J^{+}\left(x_{l},<k>\right) \geq 1$ (c'est à dire au moins un vecteur $\bar{x} \in E^{<k>}$ présente $\overline{x_{l}} \neq 0$ ) alors la présence d'une faute pour $x_{l}$ est possible mais n'est pas garantie. Cette situation est notée par In comme Incertain.

Ainsi, la détection de faute pour la transition de faute $x_{l}$ est garantie dans le cas 1 . Le dernier cas 3 exprime une incertitude sur la conclusion mais est malgré tout utile : ce cas montre la nécessité de prolonger la procédure avec plus d'informations ou alors de compléter cette procédure par une intervention humaine de maintenance préventive vérifiant l'occurrence de cet événement.

Les deux indicateurs de fautes $J^{-}\left(x_{l}, M^{<k>}\right)$ et $\left.J^{+}\left(x_{l}, M^{<k>}\right)\right)$ relatif à un marquage $M^{<k>} \in \mathcal{M}^{\text {min, }<k>}$ sont donnés par les problèmes de la programmation linéaires en nombres entiers suivant :

$$
\begin{gathered}
\left.J^{-}\left(x_{l}, M^{<k>}\right)=\min c . \bar{x} \text { (respectivement, } J^{+}\left(x_{l}, M^{<k>}\right)=\max c . \bar{x}\right) \\
\text { avec } c_{l}=1 \text { et } c_{j}=0 \text { pour } j \neq l \\
\text { sous la contrainte [3] de section } 3 \text { pour } M^{<k>} \in \mathcal{M}^{\text {min, },<k>}
\end{gathered}
$$


qui peut se résoudre par les logiciels actuels (par exemple fonction intlinprog() de Matlab). L'ensemble $\mathcal{M}^{\text {min, }<k>}$ étant déduit à l'itération $<k-1>$, il est important de noter que le diagnostic basé sur la détermination de $E^{<k>}$ à l'itération $<k>$ est remplacé par la résolution des problèmes ci-dessus qui vont sélectionner des vecteurs optimaux. On a donc évité une approche énumérative par rapport aux vecteurs d'explication éventuellement minimaux $E^{\min ,<k>}$ ce qui est un deuxième moyen de réduire la combinatoire qui se limitera aux marquages $M^{<k>} \in \mathcal{M}^{\min ,<k>}$.

\section{Discussion}

Le diagnostic proposé peut être considéré comme un module supplémentaire complétant l'approche initiale d'estimation qui alterne le calcul des marquages de base et des vecteurs d'explication minimaux. Comme le diagnostic dépend de l'estimation et non l'inverse, les deux procédures pourront être synchronisées à la fin du calcul de l'ensemble des marquages de base. Par conséquent, les opérations peuvent être réparties en deux unités fonctionnant en parallèle. L'unité proposée ne fait pas ralentir l'unité principale. Le module supplémentaire présente les avantages suivants par rapport au diagnostic de l'approche standard.

- Le calcul de tout vecteur d'explication minimal des ensembles $E^{\min ,<k>}$ pour tout marquage de base de $M^{\text {min, }<k>}$ est remplacé par la recherche de solutions optimales obtenues par programmation linéaire en nombres entiers pour chaque marquage de base de $M^{\mathrm{min},<k>}$. Par conséquent, l'aspect combinatoire est réduit. Cela implique que le calcul des ensembles $M^{\min ,<k>}$ and $E^{\min ,<k>}$ n'est pas nécessaire pour le diagnostic associé à une itération donnée $\langle k>$ même si ces ensembles sont nécessaires à l'étape suivante $<k+1>$.

- Comme le module supplémentaire opère en parallèlle avec le diagnostic classique, des états de diagnostic pourront être émis potentiellement plus tôt par le module.

- A partir de RdPs de petites tailles [BOUSSIF et al. 2017], nous pouvons constater que l'approche tabulaire [CABASINO et al. 2010] de calcul des marquages de base en déterminant l'ensemble de vecteurs d'explication minimales, est probablement de complexité exponentielle ce qui impose d'ajouter une condition de bornitude au marquage. Néanmoins, et malgré cette condition, le nombre des marquages de base peut être considérable et peut conduire à une explosion des états. Alors, un calcul en parallèle en connaissant l'ensemble des marquages initiaux au début de chaque itération sera garanti malgré l'existence d'explosion des états. Nous montrerons dans un autre article que cet avantage sera renforcé si un horizon grand est considéré.

\subsection{Indicateurs basés sur l'algorithme d'élimination de Fourier-Motzkin}

Dans le but de développer des indicateurs de fautes sous une forme algèbrique, nous pouvons aussi exploiter l'algorithme d'élimination de Fourier-Motzkin qui génère explicitement des bornes sur le nombre d'occurrence des fautes.

Le système [3] de section 3 est une inégalité matricielle linéaire de la forme $A . \bar{x} \leq b$, où $A$ est une matrice de dimension $m \times n$ à valeurs dans $\mathbb{Z}, b$ est un vecteur de dimension $m \times 1$ à valeurs dans $\mathbb{Z}$ et $\bar{x}=\left(\begin{array}{llll}\bar{x}_{1} & \overline{x_{2}} & \ldots & \bar{x}_{n}\end{array}\right)^{T}$ représente les variables de l'inégalité matricielle. Nous allons utiliser l'algorithme d'élimination de Fourier-Motzkin qui va construire un nouveau système composé d'un ensemble d'intervalles encadrant chaque variable. Comme les bornes seront fonction de variables prises dans un ordre donné, cette technique présente une analogie sur ce point avec la triangularisation effectuée par le pivot de Gauss même si aucune forme triangulaire n'apparaisse en général. 
Dans le cadre de cet article, on effectuera des éliminations hors-ligne des variables du système $A . \bar{x} \leq b$ afin de genérer des indicateurs de fautes pour un diagnostic en-ligne. Ce pré-traitement hors-ligne qu'il ne sera pas nécessaire de recommencer en-ligne, établira un ensemble d'inégalités formelles. L'analyse de ces inégalités va aussi permettre de mettre en évidence les relations internes liant les nombres de tirs des transitions et les marquages et de présenter sous un jour nouveau la dynamique du modèle qui pourra être analysée sans les matrices d'incidence et les conditions de sensibilisation d'une transition.

\subsubsection{L'algorithme d'élimination de Fourier-Motzkin}

L'algorithme d'élimination de Fourier-Motzkin est la répétition de deux étapes appliquées de la première variable $\overline{x_{1}}$ jusqu'à la dernière variable $\overline{x_{n}}$ si nous prenons arbitrairement l'ordre croissant des indices.

- Etape 1 : Calcul des intervalles

La première étape de la phase 1 est la détermination de l'intervalle qui définit la variable $x_{l}$ dont les limites sont en fonction des variables du vecteur $\overline{X_{l+1}}=\left(\begin{array}{llll}\overline{x_{l+1}} & \overline{x_{l+2}} & \ldots & x_{n}\end{array}\right)^{T}$. Permettant la manipulation des inégalités et la construction des intervalles, la normalisation des valeurs de la première colonne et la classification des inégalités conduisent à la forme matricielle $A_{l} \cdot \overline{X_{l}} \leq b_{l}$ qui est détaillée ci-dessous :

$$
\left(\begin{array}{cc}
a_{l}^{+} & A_{l}^{+} \\
-a_{l}^{-} & A_{l}^{-} \\
a_{l}^{=} & A_{l}^{=}
\end{array}\right) \cdot\left(\begin{array}{c}
\overline{x_{l}} \\
\overline{X_{l+1}}
\end{array}\right) \leq\left(\begin{array}{c}
b_{l}^{+} \\
b_{l}^{-} \\
b_{l}^{=}
\end{array}\right)
$$

Le vecteur $a_{l}^{+}$de dimension $n_{l}^{+}$contient les coefficients de $x_{l}$ qui sont tous égaux à 1 . Le vecteur $a_{l}^{-}$de dimension $n_{l}^{-}$contient les coefficients de $x_{l}$ qui sont tous égaux à -1 . Le vecteur $a_{l}^{=}$de dimension $n_{l}^{=}$contient les coefficients nuls de $x_{l}$. Les dimensions des matrices $A_{l}^{+}, A_{l}^{-}$et $A_{l}^{=}$sont $\left(n_{l}^{+} \mathrm{x} n-l\right),\left(n_{l}^{-} \times \mathrm{x}-l\right)$ et $\left(n_{l}^{=} \mathrm{x} n-l\right)$ respectivement.

Si les deux vecteurs $a_{l}^{+}$et $-a_{l}^{-}$existent, nous pouvons déterminer un intervalle pour chaque variable $\overline{x_{l}}$ dont les limites sont présentées ci-dessous :

$$
\max _{i=1, \ldots, n_{l}^{-}}\left(A_{l}^{-} \cdot \overline{X_{l+1}}-b_{l}^{-}\right)_{i} \leq \overline{x_{l}} \leq \min _{j=1, \ldots, n_{l}^{+}}\left(b_{l}^{+}-A_{l}^{+} \cdot \overline{X_{l+1}}\right)_{j}
$$

Si le vecteur $a_{l}^{+}$n'existe pas dans la matrice $A_{l}$, la limite supérieure de $\overline{x_{l}}$ est égale à $+\infty$. Si le vecteur $a_{l}^{-}$n'existe pas dans la matrice $A_{l}$, la limite inférieure de $\overline{x_{l}}$ est égale à $-\infty$.

- Etape 2 : élimination et construction d'un nouveau système

L'étape 2 consiste à éliminer la variable $\overline{x_{l}}$ du système $A_{l} \cdot \overline{X_{l}} \leq b_{l}$ avec $\overline{X_{l}}=\left(\overline{x_{l}} \overline{x_{l+1}} \ldots \overline{x_{n}}\right)^{T}$, ce qui donne un nouveau système $A_{l+1} \cdot \overline{X_{l+1}} \leq b_{l+1}$ avec pour inconnue le vecteur $\overline{X_{l+1}}=\left(\overline{x_{l+1}} \overline{x_{l+2}} \ldots\right.$ $\left.\overline{x_{n}}\right)^{T}$. La procédure d'élimination de variable $\overline{x_{l}}$ peut être faite en considérant toute les combinaisons de [5] de cette section : $\left(A_{l}^{-} \cdot \overline{X_{l+1}}-b_{l}^{-}\right)_{i} \leq\left(b_{l}^{+}-A_{l}^{+} \cdot \overline{X_{l+1}}\right)_{j}$ pour tout $i \in\left\{1, \ldots, n_{l}^{-}\right\}$, $j \in\left\{1, \ldots, n_{l}^{+}\right\}$.

Les étapes 1 et 2 de la phase 1 sont répétées jusqu'à la dernière variable $\overline{x_{n}}$. L'intervalle [ 5] de cette section associé à $\overline{x_{n}}$ prend la forme particulière suivante

$$
\max _{i=1, \ldots, n_{n}^{-}}\left(-b_{n}^{-}\right)_{i} \leq \overline{x_{n}} \leq \min _{j=1, \ldots, n_{n}^{+}}\left(b_{n}^{+}\right)_{j}
$$


qui présente une borne supérieure et une borne inférieure ne dépendant pas des variables $\overline{x_{i}}$. Au final, le résultat de la phase 1 est le système composé des inégalités [ 5] de cette section pour $\overline{x_{1}}, \overline{x_{2}}, \ldots, \overline{x_{n-1}}$ et [6] de cette section pour $\overline{x_{n}}$. L'analyse de $b^{<k>}$ montre que ces expressions sont en fonction du marquage de départ (ou de base) et de l'observation courante (pris au sens de variables) tandis que leurs formes et leurs paramètres dépendent naturellement de la structure du RdP.

\subsubsection{Géneration des indicateurs de fautes}

L'application hors-ligne de l'algorithme d'élimination de Fourier-Motzkin sur le sytème [3] de section 3 permet de génèrer des bornes sur le nombre d'occurrence des fautes : il suffira que pour chaque transition de faute, l'ordre des variables choisi soit tel que la dernière variable restante corresponde à cette transition de faute. En effet, si $\overline{x_{l}}$, associée à la transition de faute $x_{l} \in T R_{f}$, est la dernière variable, le système [6] de la section 4 donne une borne inférieure et une borne supérieure des nombres d'occurrences possibles ce qui permet de déduire pour chaque transition de faute $x_{l} \in T R_{f}$ des indicateurs de faute sous forme d'expressions algèbriques :

$$
\begin{aligned}
& J^{-}\left(x_{l}, \quad<k>\right)=\min \left\{\max _{i=1, \ldots, n_{l}^{-}}\left(-b_{l}^{-}\right)_{i}\left(\forall M^{<k>} \in \mathcal{M}^{\min ,<k>}\right)\right\} \\
& J^{+}\left(x_{l}, \quad<k>\right)=\max \left\{\min _{j=1, \ldots, n_{l}^{+}}\left(b_{l}^{+}\right)_{j}\left(\forall M^{<k>} \in \mathcal{M}^{\min ,<k>}\right)\right\}
\end{aligned}
$$

avec les paramètres $b_{l}^{-}$et $b_{l}^{+}$fonction de $M^{<k>} \in \mathcal{M}^{\text {min, }<k>}$ et de l'observation courante $y^{<k>}$.

Remarque 2. Afin d'alléger l'écriture, les formulations ci-dessus supposent que chaque problème génère une solution finie pour chaque marquage.

\subsection{Complexité}

L'approche [CABASINO et al. 2010] nécessite pour le diagnostic la génération préalable de tous les vecteurs d'explications minimaux pour l'itération courante (comme le montrent les Corollaires 4.3, 4.4 et la Proposition 4.5 ) ce qui peut être coûteux en temps : le temps d'exécution est de 227 secondes à 739 secondes pour les 4 dernières lignes du tableau IV pour le RdP Fig. 4 dans [MAHULEA et al. 2012]. A chaque fois, 34650 marquages de base sont produits. Comme les vecteurs d'explications et les marquages de base de l'approche classique [CABASINO et al. 2010] devront être produits car nécessaires aux calculs de l'itération suivante, le diagnostic proposé pourra la compléter en étant effectué dans une unité de calcul en parallèle afin de ne pas ralentir l'estimation. Le diagnostic proposé dans cet article pourra utiliser soit des indicateurs de la forme de la programmation linéaire ([2] et [3] de la section 4.1), soit les indicateurs basés sur l'algorithme de Fourier-Motzkin ([7] et [8] de la section 4.2) qui pourront être formulés facilement comme le montre l'exemple suivant.

Plus généralement, rappelons que, même si l'algorithme de Fourier-Motzkin présente une mauvaise complexité dans le pire des cas, l'algorithme du Simplex est exponentiel dans le pire des cas et nous pouvons supposer que l'algorithme classique 3.5 dans [GIUA et al. 2005] présente probablement la même limitation car il effectue une recherche en arrière dans tous les chemins possibles. D'autre part, la phase 
d'élimination de l'algorithme de Fourier-Motzkin est effectuée hors-ligne et une exécution sur une période de temps nettement plus longue qu'une itération du diagnostic n'est pas rédhibitoire. Nous avons $O\left(((|P|+n) / 4)^{2^{n}}\right)$ pour la phase hors-ligne (rappelons que $\left.n=\left|T R_{n o}\right|\right)$. Ainsi, la complexité ne dépend pas du nombre de marquages et de la magnitude des marquages considérés ce qui constitue un avantage pour la phase hors-ligne. Nos essais sur les exemples tirés de la littérature montrent que fréquemment l'algorithme de Fourier-Motzkin s'applique sans difficulté car souvent les matrices d'incidence observables ne sont pas pleines : elles présentent de nombreux coefficients nuls. De plus, elles peuvent être décomposées en sous-structures indépendantes comme dans l'exemple suivant. Pour la phase en-ligne, la complexité pour chaque itération est $O\left(q \cdot\left|\mathcal{M}^{\min ,<k>}\right| . n\right)$ où $q$ est le nombre de termes contenus dans les bornes. Elle est donc polynomiale en fonction du nombre de transitions non-observables et des marquages de base à condition que la détermination de ces derniers soit réalisée.

\section{Exemple}

Nous considérons une étude de cas présentée dans [MAHULEA et al. 2012], qui peut être facilement trouvée sur le web. La Figure 1 est un RdP étiqueté qui modélise un sous-système de fabrication automatisé. Pour le RdP de la figure 1, les ensembles des transitions observables et non-observables sont $T R_{o b}=$

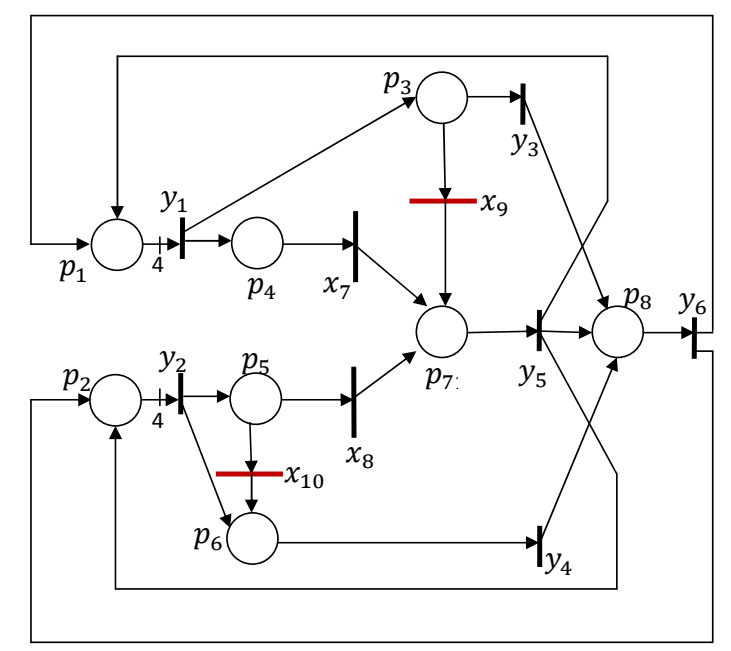

Figure 1. Modèle RdP étiqueté d'un sous-système de fabrication

$\left\{y_{1}, y_{2}, y_{3}, y_{4}, y_{5}, y_{6}\right\}$ et $T R_{n o}=\left\{x_{7}, x_{8}, x_{9}, x_{10}\right\}$ respectivement. Rappelons que $M^{<1>}=M^{\text {init }}$ est le marquage initial. Pour alléger la notation, nous notons ci-dessous $M^{<k>}=M$ et $M_{i}^{<k>}=M\left(p_{i}\right)=M_{i}$ ce qui est le marquage de départ de la place $p_{i}$ pour l'itération $\langle k\rangle$. De même, $\overline{x_{7}}, \overline{x_{8}} \overline{x_{9}}$, et $\overline{x_{10}}$ expriment les nombres de tirs à estimer des transitions $x_{7}, x_{8}, x_{9}$ et $x_{10}$ respectivement. Par hypothèse, $\overline{y_{1}}, \overline{y_{2}}, \overline{y_{3}}, \overline{y_{4}}, \overline{y_{5}}$ et $\overline{y_{6}}$ ne peuvent prendre que 2 valeurs, 0 ou 1 et pour chaque itération si $\overline{y_{i}}=1$, alors $\overline{y_{j}}=0$, pour tout $j \neq i$. Les transitions observables de l'ensemble $T R_{o b}=\left\{y_{1}, y_{2}, y_{3}, y_{4}, y_{5}, y_{6}\right\}$ sont étiquetées comme suit : $\ell\left(y_{1}\right)=a, \ell\left(y_{2}\right)=b, \ell\left(y_{3}\right)=c, \ell\left(y_{4}\right)=d, \ell\left(y_{5}\right)=e, \ell\left(y_{6}\right)=f$. Nous considérons l'observation $w=$ abedd associée à la séquence unique observée $y_{1} y_{2} y_{5} y_{4} y_{4}$ à partir du marquage initial $(20,20,0,0,0,0,0,0)^{T}$. 
Génération hors-ligne des indicateurs de fautes :

Le polyèdre [3] de la section 3 devient

$$
\left(\begin{array}{rrrr}
0 & 0 & 1 & 0 \\
1 & 0 & 0 & 0 \\
0 & 1 & 0 & 1 \\
0 & 0 & 0 & -1 \\
-1 & -1 & -1 & 0
\end{array}\right) \cdot\left(\begin{array}{l}
\overline{x_{7}} \\
\overline{x_{8}} \\
\overline{x_{9}} \\
\overline{x_{10}}
\end{array}\right) \leq\left(\begin{array}{l}
M_{3}-\overline{y_{3}} \\
M_{4} \\
M_{5} \\
M_{6}-\overline{y_{4}} \\
M_{7}-\overline{y_{5}}
\end{array}\right) \text { avec } \overline{x_{7}}, \overline{x_{8}}, \overline{x_{9}}, \overline{x_{10}} \geq 0 .
$$

- Pour l'isolation de faute $\overline{x_{10}}$, nous choisissons l'ordre d'élimination $\overline{x_{7}}, \overline{x_{8}}, \overline{x_{9}}, \overline{x_{10}}$.

L'élimination de $\overline{x_{7}}$ se fait à partir de deuxième et dernière ligne qui donnent $\overline{x_{7}} \leq M_{4}$ et $-\overline{x_{8}}-\overline{x_{9}}-$ $M_{7}+\overline{y_{5}} \leq \overline{x_{7}}$. De plus, $0 \leq \overline{x_{7}}$. Ainsi, nous obtenons l'encadrement de $\overline{x_{7}}$ qui est $\max \left(0,-\overline{x_{8}}-\right.$ $\left.\overline{x_{9}}-M_{7}+\overline{y_{5}}\right) \leq \overline{x_{7}} \leq M_{4}$ et nous devons aussi garder les lignes 1,3 et 4 du polyèdre.

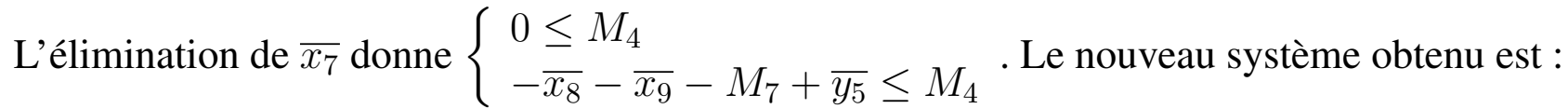

$$
\left(\begin{array}{rrr}
-1 & -1 & 0 \\
0 & 1 & 0 \\
1 & 0 & 1 \\
0 & 0 & -1
\end{array}\right) \cdot\left(\begin{array}{l}
\overline{x_{8}} \\
\overline{x_{9}} \\
\overline{x_{10}}
\end{array}\right) \leq\left(\begin{array}{l}
M_{4}+M_{7}-\overline{y_{5}} \\
M_{3}-\overline{y_{3}} \\
M_{5} \\
M_{6}-\overline{y_{4}}
\end{array}\right) \text { avec } \overline{x_{8}}, \overline{x_{9}}, \overline{x_{10}} \geq 0 .
$$

La même procédure se repète pour l'élimination successive de $\overline{x_{8}}, \overline{x_{9}}, \overline{x_{10}}$. Finalement, nous obtenons le système d'inégalités suivant :

$$
\left\{\begin{array}{l}
\max \left(0,-\overline{x_{8}}-\overline{x_{9}}-M_{7}+\overline{y_{5}}\right) \leq \overline{x_{7}} \leq M_{4} \\
\left.\max \left(0,-\overline{x_{9}}-M_{4}\right)-M_{7}+\overline{y_{5}}\right) \leq \overline{x_{8}} \leq-\overline{x_{10}}+M_{5} \\
\max \left(0, \overline{x_{10}}-M_{4}-M_{5}-M_{7}+\overline{y_{5}}\right) \leq \overline{x_{9}} \leq M_{3}-\overline{y_{3}} \\
\max \left(0,-M_{6}+\overline{y_{4}}\right) \leq \overline{x_{10}} \leq \min \left(M_{5}, M_{3}+M_{4}+M_{5}+M_{7}-\overline{y_{3}}-\overline{y_{5}}\right)
\end{array}\right.
$$

La dernière relation de 2 donne les indicateurs de fautes générés hors-ligne.

$$
\begin{aligned}
& J^{-}\left(x_{10},<k>\right)=\min \left\{\max \left(0, \overline{y_{4}}-M_{6}\right)\left(\forall M^{<k>} \in \mathcal{M}^{\min ,<k>}\right)\right\} \\
& J^{+}\left(x_{10},<k>\right)=\max \left\{\min \left(M_{5}, M_{3}+M_{4}+M_{5}+M_{7}-\overline{y_{3}}-\overline{y_{5}}\right)\left(\forall M^{<k>} \in \mathcal{M}^{\min ,<k>}\right)\right\}
\end{aligned}
$$

- Pour l'isolation de faute $\overline{x_{9}}$, nous choisissons l'ordre d'élimination $\overline{x_{7}}, \overline{x_{8}}, \overline{x_{10}}, \overline{x_{9}}$ et nous obtenons :

De même,

$$
\left\{\begin{array}{l}
\max \left(0,-\overline{x_{8}}-\overline{x_{9}}-M_{7}+\overline{y_{5}}\right) \leq \overline{x_{7}} \leq M_{4} \\
\left.\max \left(0,-\overline{x_{9}}-M_{4}\right)-M_{7}+\overline{y_{5}}\right) \leq \overline{x_{8}} \leq-\overline{x_{10}}+M_{5} \\
\max \left(0,-M_{6}+\overline{y_{4}}\right) \leq \overline{x_{10}} \leq \min \left(M_{5}, \overline{x_{9}}+M_{4}+M_{5}+M_{7}-\overline{y_{5}}\right) \\
\max \left(0,-M_{4}-M_{5}-M_{7}+\overline{y_{5}},-M_{4}-M_{5}-M_{6}-M_{7}+\overline{y_{4}}+\overline{y_{5}}\right) \leq \overline{x_{9}} \leq M_{5}
\end{array}\right.
$$

$$
\begin{aligned}
& J^{-}\left(x_{9},<k>\right)=\min \left\{\max \left(0, \overline{y_{4}}+\overline{y_{5}}-M_{4}-M_{5}-M_{6}-M_{7}, \overline{y_{5}}-M_{4}-M_{5}-M_{7}\right)\right. \\
& \left.\quad\left(\forall M^{<k>} \in \mathcal{M}^{\min ,<k>}\right)\right\} \\
& J^{+}\left(x_{9},<k>\right)=\max \left\{M_{3}-\overline{y_{3}}\left(\forall M^{<k>} \in \mathcal{M}^{\min ,<k>}\right)\right\}
\end{aligned}
$$

\section{Détection en-ligne des fautes :}

La mise à jour de l'ensemble des marquages initiaux, qui sont aussi l'ensemble des marquages de base, pour chaque observation élémentaire est réalisée en se basant sur l'algorithme tabulaire de calcul des vecteurs d'explication minimaux [CABASINO et al. 2010].

Dans le tableau 1, les résultats de diagnostic sont présentés pour les deux approches : l'approche basée 
sur la résolution des problèmes PLNE et l'approche basée sur l'application hors-ligne de l'algorithme d'élimination de Fourier-Moutzkin. En utilisant Matlab, les calculs sont effectués sur un PC Intel avec une horloge de $1.80 \mathrm{Ghz}$. Les trois états de diagnostic, notés $N$, In, et $F$, correspondent respectivement à un état Normal, un état Incertain et un état de Faute. Nous notons $\Delta_{1}$ et $\Delta_{2}$ les états de diagnostic respectivement des transitions des fautes $x_{9}$ et $x_{10}$. Le tableau 5.1 montre que la faute $x_{10}$ est détectée à

Tableau 5.1. Résultats de diagnostic

\begin{tabular}{|c|c|c|c|c|c|c|c|c|}
\hline \multirow[t]{2}{*}{$k$} & \multirow[t]{2}{*}{$y^{<k>}$} & \multirow[t]{2}{*}{$\begin{array}{l}\text { Marquages initiaux } \\
\qquad M^{<k>}\end{array}$} & \multicolumn{3}{|c|}{$\begin{array}{l}\text { Approche basée } \\
\text { sur des problèmes PLNE }\end{array}$} & \multicolumn{3}{|c|}{$\begin{array}{c}\text { Approche basée } \\
\text { sur Fourier Moutzkin }\end{array}$} \\
\hline & & & $\begin{array}{c}\text { Indicateur de } \\
\text { faute }\end{array}$ & $\left(\Delta_{1}, \Delta_{2}\right)$ & $\begin{array}{c}\text { Temps } \\
\text { d'exécution }(s) \\
\end{array}$ & $\begin{array}{c}\text { Indicateur de } \\
\text { faute }\end{array}$ & $\left(\Delta_{1}, \Delta_{2}\right)$ & $\begin{array}{c}\text { Temps } \\
\text { d'exécution }(s) \\
\end{array}$ \\
\hline 0 & $\varepsilon$ & $(20,20,0,0,0,0,0)^{T}$ & $\begin{aligned} J^{-}\left(x_{9}\right) & =0 ; J^{+}\left(x_{9}\right)=0 \\
J^{-}\left(x_{10}\right) & =0 ; J^{+}\left(x_{10}\right)=0\end{aligned}$ & $(N, N)$ & 2.327 & $\begin{array}{c}J^{-}\left(x_{9}\right)=0 ; J^{+}\left(x_{9}\right)=0 \\
J^{-}\left(x_{10}\right)=0 ; J^{+}\left(x_{10}\right)=0\end{array}$ & $(N, N)$ & 0.0013 \\
\hline 2 & $t_{1} t_{2}$ & $(16,20,1,1,0,0,0,0)^{T}$ & $\begin{array}{c}J^{-}\left(x_{9}\right)=0 ; J^{+}\left(x_{9}\right)=1 \\
J^{-}\left(x_{10}\right)=0 ; J^{+}\left(x_{10}\right)=0\end{array}$ & $(\operatorname{In}, N)$ & 1.780 & $\begin{array}{c}J^{-}\left(x_{9}\right)=0 ; J^{+}\left(x_{9}\right)=1 \\
J^{-}\left(x_{10}\right)=0 ; J^{+}\left(x_{10}\right)=0\end{array}$ & $(I n, N)$ & 0.0009 \\
\hline 3 & $t_{1} t_{2} t_{5}$ & $(16,16,1,1,1,1,0,0)^{T}$ & $\begin{aligned} J^{-}\left(x_{9}\right) & =0 ; J^{+}\left(x_{9}\right)=1 \\
J^{-}\left(x_{10}\right) & =0 ; J^{+}\left(x_{10}\right)=1\end{aligned}$ & $(I n, I n)$ & 1.905 & $\begin{array}{c}J^{-}\left(x_{9}\right)=0 ; J^{+}\left(x_{9}\right)=1 \\
J^{-}\left(x_{10}\right)=0 ; J^{+}\left(x_{10}\right)=1\end{array}$ & $($ In, In $)$ & 0.0049 \\
\hline 5 & $\left|t_{1} t_{2} t_{5} t_{4} t_{4}\right|$ & $\begin{array}{l}(17,17,0,1,1,0,0,1)^{T} \\
(17,17,1,0,1,0,0,1)^{T}\end{array}$ & $\begin{array}{c}J^{-}\left(x_{9}\right)=0 ; J^{+}\left(x_{9}\right)=1 \\
J^{-}\left(x_{10}\right)=1 ; J^{+}\left(x_{10}\right)=1\end{array}$ & $(I n, F)$ & 1.700 & $\begin{array}{c}J^{-}\left(x_{9}\right)=0 ; J^{+}\left(x_{9}\right)=1 \\
J^{-}\left(x_{10}\right)=1 ; J^{+}\left(x_{10}\right)=1\end{array}$ & $(I n, F)$ & 0.0008 \\
\hline
\end{tabular}

l'itération $<5>$. On observe aussi que le temps de calcul des indicateurs générés par l'approche d'élimination de Fourier-Moutkin est fortement réduit par rapport aux indicateurs générés par la programmation linéaire en nombres entiers.

\section{Conclusion}

Dans cet article, nous avons proposé des indicateurs de diagnostic d'abord sous la forme de problèmes de la programmation linéaire puis en termes d'expressions algèbriques grâce à une adaptation de l'algorithme d'élimination de Fourier-Motzkin. Ils constituent les éléments d'un module de diagnostic qui peut s'insérer en parallèle du module d'estimation de l'approche classique dans un calcul distribué. Notons que l'approche proposée autorise un diagnostic partiel localisé à un sous-réseau de Petri. Bien que la complexité de la phase hors-ligne basée sur l'algorithme d'élimination de Fourier-Motzkin soit mauvaise dans le pire des cas, la complexité de son application en-ligne est polynomiale pour chaque itération. Les tests numériques montrent un temps d'exécution réduit ce qui permet un diagnostic anticipé dans l'itération courante par rapport à la technique devant attendre la fin de la génération de tous les vecteurs d'explication. La complexité est ainsi une donnée connue ce qui est un avantage.

Notons que les inégalités [5] et [6] de la section 4 effectuent un encadrement des nombres de tirs avec des bornes qui sont des relations $(\min , \max ,+)$. Les termes de base qui sont du type $(\max ,+)$ ou $(\min ,+)$ montrent explicitement les liens entre les nombres de tirs des transitions et les marquages des places. Les indicateurs indiquent non seulement la présence éventuelle d'une faute mais aussi la fréquence de cette faute à chaque observation. Plus encore, l'analyse hors-ligne de ces indicateurs permet d'anticiper sur leurs efficacités en-ligne comme l'illustrent les exemples et une perspective est de développer cet aspect. 


\section{Bibliographie}

Ahmed A.,Behzad B., Fourier-Motzkin method for failure diagnosis in Petri Net models of discrete event systems. p. 165-170, Wodes 2016.

Basile F., Cabasino M. P., Seatzu C., State estimation and fault diagnosis of labeled time petri net systems with unobservable transitions. IEEE Transactions on Automatic Control, 2014, vol. 60, no 4, p. 997-1009.

Berthomieu B., Diaz M., Modeling and verification of time dependent systems using time Petri nets. IEEE transactions on software engineering, 1991, vol. 17, no 3, p. 259-273.

Boussif A., LiU B., Ghazel M., An experimental comparison of three diagnosis techniques for discrete event systems. In : DX'17-28th International Workshop on Principles of Diagnosis. 2017. p. p8.

Boussif A., GHAZEL M., KLAI K., DPN-SOG : A software tool for fault diagnosis of labeled Petri nets using the semisymbolic diagnoser. In : 11ème Colloque sur la Modélisation des Systèmes Réactifs (MSR 2017). 2017.

CABAsino M., Giua A., Seatzu C., Fault detection for discrete event systems using Petri nets with unobservable transitions. Automatica, vol. 46, no 9, p. 1531-1539, 2010.

Cabasino M. P., Giua A., Paoli A., Seatzu C., Decentralized diagnosis of discrete-event systems using labeled Petri nets. IEEE Transactions on Systems, Man, and Cybernetics : Systems, 2013, vol. 43, no 6, p. 1477-1485.

Chouchane A., Declerck P., Khedher A., Kamoun A., Diagnostic based on estimation using linear programming for partially partially observable petri nets with indistinguishable events. International Journal of Systems Science : Operations and Logistics, 2018, p. 1-14.

Genest B., Kuske D., Muscholl A., A Kleene theorem and model checking algorithms for existentially bounded communicating automata. Information and Computation, 2006, vol. 204, no 6, p. 920-956.

GiUa A., Seatzu C., Fault detection for discrete event systems using Petri nets with unobservable transitions. In Proc. 44th IEEE Conf. on Decision and Control, pages 6323-6328, December 2005.

GiuA A., State Estimation and Fault Detection Using Petri Nets. Applications and Theory of Petri Nets : Proc. 32nd Int. Conf. on Applications and Theory of Petri nets 2008 (Newcastle, UK), June 20-24, 2011. Lecture Notes in Computer Science Vol. 6709, pp. 419-428, L.M. Kristensen and L. Petrucci (Eds.) Springer-Verlag, 2011.

Jiroveanu G., Boel R. K., Bordbar B., On-line monitoring of large Petri net models under partial observation. Discrete Event Dynamic Systems, 2008, vol. 18, no 3, p. 323-354.

LefEbVRE D., Diagnosis with Petri nets according to partial events and states observation. 8th IFAC Symposium on Fault Detection, Safeprocess, P. 1244-1249, Mexico, 2012.

Mahulea C., Seatzu C., Cabasino M., Silva M., Fault Diagnosis of Discrete-Event Systems Using Continuous Petri Nets. IEEE Transactions on Systems, Man, and Cybernetics, Vol.42, pp 970-984, 2012.

Ran N., Wang S., Su H., WAng C., Fault diagnosis for discrete event systems modeled by bounded Petri nets. Asian Journal of Control, 2017, vol. 19, no 4, p. 1532-1541.

RU Y., HADJICOSTIS C.N., Bounds on the number of markings consistent with label observations in Petri nets. IEEE Transactions on Automation Science and Engineering, 2009, vol. 6, no 2, p. 334-344.

STREMERSCH G., Boel R.K., Structuring acyclic Petri nets for reachability analysis and control. Discrete Event Dynamic Systems, 2002, vol. 12, no 1, p. 7-41.

Sun Z., LiaO T., LiU K., JiAng L., Kim J. H., Dou S. X., Fly-Eye Inspired Superhydrophobic Anti-Fogging Inorganic Nanostructures. Small , 2014, vol. 10, no 15, p. 3001-3006. 